\title{
Downregulation of VMP1 confers aggressive properties to colorectal cancer
}

\author{
XIAN-ZHI GUO $^{1 *}$, XIAO-LEI YE ${ }^{2 *}$, WEI-ZHONG XIAO ${ }^{3 *}$, XUE-NI WEI $^{6}$, QING-HUA YOU ${ }^{4}$, XIAO-HANG CHE ${ }^{2}$, \\ YAN-JUN CAI $^{3}$, FANG CHEN ${ }^{7}$, HAO YUAN $^{5}$, XIAO-JIAN LIU $^{1}$ and MING-HUA YU ${ }^{1}$ \\ ${ }^{1}$ Department of Medical Oncology, Shanghai Pudong Hospital, Fudan University Pudong Medical Center, Pudong, \\ Shanghai 201399; ${ }^{2}$ Drugs and Pharmacology Laboratory, Ningbo Institute of Medical Sciences, Ningbo, Zhejiang 315020; \\ Departments of ${ }^{3}$ Internal Neurology, ${ }^{4}$ Pathology and ${ }^{5}$ General Surgery, Shanghai Pudong Hospital, Fudan University \\ Pudong Medical Center, Pudong, Shanghai 201399; ${ }^{6}$ Department of Pharmacology, China Pharmaceutical University, \\ Nanjing, Jiangsu 211198; ${ }^{7}$ Department of Hematology, Nanfang Hospital, Southern Medical University, \\ Guangzhou 510515, P.R. China
}

Received July 16, 2015; Accepted August 7, 2015

DOI: 10.3892/or.2015.4240

\begin{abstract}
Vacuole membrane protein 1 (VMP1) was recently found to be involved in the process of tumor metastasis and is also considered to play a vital role in balancing apoptosis and autophagy. In the present study, the expression of VMP1 in colorectal cancer and matched adjacent non-cancerous tissues was evaluated by immunohistochemistry (IHC) for studying the role of VMP1 in the process of colorectal cancer. Kaplan-Meier analysis and the log-rank test were used to calculate the correlation of classic clinicopathological characteristics related to survival and the expression of VMP1. In vitro, a VMP1 stable gene silencing cell model was constructed using a lentiviral vector. The invasive ability and proliferation of colorectal cancer cells were evaluated by Transwell and MTT assays, respectively, and the underlying signaling pathway was explored by western blotting. Additionally, drug susceptibility to cisplatin, oxaliplatin and 5-FU was tested before and after VMP1 knockout. Finally, an animal model was constructed to explore the role of VMP1 in the physiopathologic process of colorectal cancer. Our results indicated that VMP1 showed increased expression in the adjacent non-cancer tissues compared with that in the colorectal cancer tissues. For different stages of colorectal cancer, expression of VMP1 had
\end{abstract}

Correspondence to: Professor Xiao-Jian Liu or Professor Ming-Hua $\mathrm{Yu}$, Department of Medical Oncology, Shanghai Pudong Hospital, Fudan University Pudong Medical Center, 2800 Gongwei Road, Pudong, Shanghai 201399, P.R. China

E-mail: 1xj068@hotmail.com

E-mail:ymh3011@hotmail.com

*Contributed equally

Key words: vacuole membrane protein 1 colorectal cancer, lentivirus, invasion, proliferation, chemotherapy a negative correlation with the malignancy of the cancer. In clinical research, we also found that the median survival of patients with low VMP1 expression was much shorter than the survival of patients with high expression. In vitro, after infection with the lentivirus, cells with VMP1 knockout gained significant aggressive properties in regards to invasion and proliferation, and the mechanisms may be related to the activation of the PI3K/Akt/ZO-1/E-cadherin pathway. We also found that shVMP1 cells were more sensitive to 5-FU, but not cisplatin and oxaliplatin. Finally, we found a higher number of formed nodules in nude mice after intraperitoneal injection with shVMP1 cells in the in vivo study.

\section{Introduction}

Colorectal cancer is one of the most common malignancies in developing countries. Some patients exhibit a poor prognosis due to resistance to chemotherapy and other treatments. The underlying cause may be the enhanced viability of tumor cells. In the life cycle of tumor cells, cells undergo a condition of inadequate blood supply, and the ensuing absence of oxygen and glucose deprivation may result in autophagy (1). In recent years, autophagy has been attracting extensive attention as a common event caused by ambient pressure or cellular stress. In detail, autophagy is a general term for the degradation of cytoplasmic components within lysosomes, producing amino acid, saccharides, and nucleotides to suffice the physiological processes of cells which lack nutrients in turn reducing the stress from apoptosis (2). However, it serves as only a temporary relief for the survival of cells in poor condition, since persistence of this process often results in cell death (3).

The expression of several specific proteins is often accompanied by the process of autophagy, which can be recognized as biomarkers related to the prognosis of colorectal cancer. These proteins are specifically expressed in tumor cells but not in normal cells. These include light chain 3 (LC3), Beclin-1, p62 and GABA-A receptor-associated protein (GABARAP). LC3 accumulating around the nucleus is closely related 
with the existence of autophagy as well as indicative of a better prognosis of colon cancer (4). In addition, in advanced colorectal cancer, Beclin-1 is significantly highly expressed in situ compared with metastatic sites and also indicates a better prognosis (5). In contrast, overexpression of GABARAP in colorectal cancer cells results in poor differentiation and reduced patient survival (6). Another study also indicated that detection of the combination of the above biomarkers facilitated prediction of prognosis in patients receiving 5-FU treatment (7).

The prognosis of colorectal cancer depends on the occurrence of tumor metastasis. Yet, the relationship between autophagy and metastasis is still elusive (8). It is generally considered that autophagy helps in reducing necrosis and inflammation, preventing metastasis at the early stage of cancer. Yet, from another point of view, it may also help in providing higher survival probability for tumor cells circulated in blood and lymph nodes (9).

Vacuole membrane protein 1 (VMP1) is a transmembrane protein which has been shown to be located mainly in the Golgi apparatus and endoplasmic reticulum. It was first observed in pancreatitis, participating in the process of autophagy (10). Subsequent study of tumor cells demonstrated that it also plays a key role in the development of tumors in processes such as proliferation (11), autophagy (12), metastasis (11) and drug sensitivity (13). A recent study on VMP1 showed that colorectal cancer cells initiated the process of autophagy under the stimulation of rapamycin or lack of nutrients with an increased expression of VMP1 (14). However, this phenomenon was diminished by siRNA interference of VMP1. Further study indicated that colorectal cancer cells also became more sensitive to etoposide and tended to undergo apoptosis under starvation. In this previous study, VMP1 was considered as a key regulator of autophagy. Namely active VMP1 promoted the process of autophagy, maintained the survival of tumor cells as well as was insensitive to apoptotic signaling. VMP1 could be regarded as a suitable biomarker for colorectal cancer. However, this study appeared to be limited owing to the lack of detection of VMP1 in clinical samples as well as that etoposide is not the first-line drug in treating patients with colorectal cancer.

Therefore, it is important to explore the effect of VMP1 on the biological characteristics of colorectal cancer cells and thus elucidate the underlying mechanisms, clarifying the implications of VMP1 in the prognosis of patients suffering from colorectal cancer. In the present study, expression of VMP1 was detected in clinical samples as well as different colorectal cancer cell lines to establish relationships between VMP1 and the prognosis of colorectal cancer. Furthermore, an shRNA lentiviral vector was constructed to silence the VMP1 gene to observe heritable changes in the cell lines which originally highly express VMP1. The present study focused on the proliferation, drug sensitivity, metastasis as well as the signaling pathways related to the processes mentioned above.

\section{Materials and methods}

Cell culture. The colorectal cell lines HT-29, SW620, RKO and LOVO were obtained from Dr Zeng (State Key Laboratory of Oncology in Southern China, Sun Yat-sen University Cancer
Center, Guangzhou, China) and were grown in RPMI-1640 medium (Invitrogen, Carlsbad, CA, USA) supplemented with 10\% fetal bovine serum (FBS). 293T and SW480 cell lines were purchased from the Shanghai Institute of Cell Biology (Shanghai, China). 293T and SW480 cells were separately cultured in Dulbecco's modified Eagle's medium (DMEM) and RPMI-1640 medium with $10 \% \mathrm{FBS}$ at $37^{\circ} \mathrm{C}$ in a humidified atmosphere of $5 \% \mathrm{CO}_{2}$.

Patient data. Paraffin-embedded, archived colorectal cancer samples obtained from 94 patients were histologically and clinically diagnosed at Fudan University Pudong Medical Center between 2007 and 2009. Of the 94 colorectal cancer tissues, 52 matched adjacent non-cancerous tissues were used as controls. Prior to the use of these clinical materials for investigation, informed consent from patients and approval from the Institute Research Ethics Committee were obtained. Primary cancers of the colorectum were classified according to the pathological TNM classification (15). Clinical information of the samples is described in detail in Table I. Patients included 47 males and 47 females, with ages ranging from 24 to 85 years (mean, 65.4 years). The data for metastasis pertain to its presence at any time during the follow-up. The median follow-up time for overall survival was 59.0 months for patients still alive at the time of analysis and ranged from 6 to 86 months. A total of $39(41.5 \%)$ patients died during follow-up.

VMP1 shRNA construction and lentiviral production. Three target sequences for VMP1 mRNA were chosen according to the RNAi Consortium (TRC) shRNA Library (Broad Institute) (16). The VMP1 shRNA single-strand oligonucleotides were: 1F, 5'-CCGGGTGCTTATAGCTACGTATTATC TCGAGATAATACGTAGCTATAAGCACTTTTTG-3' and 1R, 5'-AATTCAAAAAGTGCTTATAGCTACGTATTATCT CGAGATAATACGTAGCTATAAGCAC-3'; 2F, 5'-CCGGCA ACAGTATGTGCAACGTATACTCGAGTATACGTTGCAC ATACTGTTGTTTTTG-3' and 2R, 5'-AATTCAAAAACAA CAGTATGTGCAACGTATACTCGAGTATACGTTGCACA TACTGTTG-3'; 3F, 5'-CCGGGCAATGAACAAGGAACAT CATCTCGAGATGATGTTCCTTGTTCATTGCTTTTTG-3' and 3R, 5'-AATTCAAAAAGCAATGAACAAGGAACATC ATCTCGAGATGATGTTCCTTGTTCATTGC-3'.

Two complementary single-strand oligonucleotides containing the target sequences were synthesized (Shanghai, China) chemically and annealed. The double-stranded oligonucleotides were inserted between the AgeI and EcoRI restriction sites in the pLKO-green fluorescent protein (GFP) small interfering RNA (siRNA) vector that contains cytomegalovirus-driven enhanced green fluorescent protein (EGFP) reporter gene. The ligated plasmid was transformed into E. coli DH5 $\alpha$ competent cells for plasmid amplification. The plasmids from positive colonies were identified by RT-PCR and DNA sequencing.

Recombinant lentiviruses were produced by co-transfecting 293T cells with three combinant lentiviral vectors, $\Delta 8.91$ and pVSV-G (10:10:1) using the cationic lipid complex method (X-tremeGENE HP DNA Transfection Reagent; Roche). The culture supernatants containing the produced viruses were harvested at $48 \mathrm{~h}$ after transfection, and concentrated by centrifugation at $4,000 \mathrm{x}$ at $4^{\circ} \mathrm{C}$ for $10-15 \mathrm{~min}$. SW480 cells 
were subcultured at a density of $1 \times 10^{6}$ cells/well. Cells were divided into 4 groups: CON (infected with negative control lentiviral vector) and VMP1 shRNA 1-3 (infected with the VMP1 lentiviral vectors). SW480 cells were plated into 6-well dishes at $5 \times 10^{5}$ cells/well. The next day, the cells were infected with the same viral titer with $2 \mu \mathrm{g} / \mathrm{ml}$ Polybrene. After $24 \mathrm{~h}$ post-infection, the media were replaced with media containing $2 \mu \mathrm{g} / \mathrm{ml}$ puromycin. Cells were maintained and allowed to grow for 7-9 days; media were replaced without puromycin and then passaged for follow-up assays.

Western blot analysis. Equal numbers of cells were lysed in lysis buffer composed of 0.6 M Tris- $\mathrm{HCl}$ (pH 6.8), 10\% SDS and protease inhibitor cocktail. Samples were incubated at $4^{\circ} \mathrm{C}$ for $10 \mathrm{~min}$, and then centrifuged at $10,000 \mathrm{x} \mathrm{g}$ for $15 \mathrm{~min}$ at $4^{\circ} \mathrm{C}$. The supernatants were transferred, mixed and boiled in sample buffer. The supernatants were separated by polyacrylamide gel electrophoresis and transferred to a PVDF membrane (Bio-Rad, Hercules, CA, USA). Then incubation of the membrane was carried out at room temperature in blocking buffer consisting of $5 \%$ fat-free milk dissolved in $1 \mathrm{X}$ TBST (10 mM Tris-base, pH 7.5, $100 \mathrm{mM} \mathrm{NaCl}$ and 1\% Tween-20) for $1 \mathrm{~h}$ followed by incubation with the blocking buffer containing the primary antibody, such as anti-VMP1, anti-AKT, anti-pAKT, anti-ZO-1 (Cell Signaling Technology, Danvers MA, USA), anti-E-cadherin or anti- $\beta$-actin (BD, Shanghai, China) at $4^{\circ} \mathrm{C}$ overnight. The membrane was washed with TBS-T, and incubated with the secondary antibody for $1 \mathrm{~h}$. The blot was exposed to ECL (Amersham) after TBS washing.

Cell proliferation experiment by MTT method. Cells of the SW480 CON group (infected with the negative control lentiviral vector) and the SW480 VMP1 shRNA1 group (infected with the VMP1 lentiviral vector) were seeded into a 96-well microplate at a density of $5 \times 10^{3}$ cells/well and incubated overnight in $10 \%$ FBS medium. Each group had 3 wells. After incubation for $12,24,48$ and $72 \mathrm{~h}$ at $37^{\circ} \mathrm{C}$, MTT reagent $(5 \mathrm{mg} / \mathrm{ml})$ in phosphate-buffered saline (PBS) was added to the cells $(10 \mu \mathrm{l} /$ well), and the cells were incubated for $4 \mathrm{~h}$ at $37^{\circ} \mathrm{C}$. The supernatant was discarded and $200 \mu \mathrm{l}$ of dimethylsulfoxide (DMSO) was added to each well to solubilize the MTT-formazan product. Finally, samples were measured on a multi-well spectrophotometer (Thermo, USA) at a test wavelength of $560 \mathrm{~nm}$ with a reference wavelength of $650 \mathrm{~nm}$.

Drug sensitivity experiment. Cells were seeded at 5,000 cells/well into 96 -well plates (200 $\mu \mathrm{l} /$ well) and allowed to grow for $24 \mathrm{~h}$ before treatment with the complex. Different concentrations of cisplatin, 5-FU and oxaliplatin were added into the cells; each group had 3 wells. After a further $24 \mathrm{~h}$, MTT reagent $(5 \mathrm{mg} / \mathrm{ml})$ in PBS was added to the cells $(10 \mu \mathrm{l} /$ well) and incubated for $4 \mathrm{~h}$ at $37^{\circ} \mathrm{C}$. Two hundred microliters of DMSO was added to each well to solubilize the MTT-formazan product after removal of the medium. Samples were measured on a multi-well spectrophotometer (Thermo) at a test wavelength of $595 \mathrm{~nm}$ and a reference of $650 \mathrm{~nm}$.

Matrigel-based invasion assay. Assays were performed in Transwell chambers (Corning Inc., USA) with an $8-\mu \mathrm{m}$ pore size coated with Matrigel (BD, USA). SW480 cells infected with the scrambled shRNA and VMP1 shRNA1 were trypsinized and suspended in $1 \%$ FBS. After counting, the cells were plated into the upper chamber and $600 \mu 1$ medium supplemented with $10 \% \mathrm{FBS}$ was placed into the lower chamber. After incubation at $37^{\circ} \mathrm{C}$ for $6 \mathrm{~h}$, cells on the upper surface of the filters were removed and cells adhering to the undersurface of the filter membrane were dyed with $0.5 \%$ crystal violet for $30 \mathrm{~min}$. The crystal violet was washed with PBS for 3 times. Cells on the lower chamber were counted under a microscope in four fields randomly. The mean cell numbers were recorded and analyzed. The experiment was repeated 3 times.

Immunohistochemistry (IHC). IHC was carried out to study altered protein expression in 94 human colorectal cancer tissues and 52 matched adjacent non-cancerous tissues. A commercially available antibody against VMP1 (1:100 lot ab116006, rabbit polyclonal immunoglobulin G; Abcam Biotechnology, USA) was used as the primary antibody. An immunohistochemical kit (SP-9001 rabbit SP kit, lot 50581654) was obtained from Zhongshan Golden Bridge Biotechnology Co., Ltd. (Beijing, China). For each sample, one score was given according to the percent of positive cells as: no positive cells, $0 ;<5 \%$ positive cells, 1 point; $5-35 \%$ positive cells, 2 points; $36-70 \%$ positive cells, 3 points; $>70 \%$ positive cells, 4 points. To achieve objectivity, the intensity of positive staining was also used in a four-tier scoring system: 0 (negative staining), 1 (weak staining exhibited as light yellow), 2 (moderate staining exhibited as yellow brown), and 3 (strong staining exhibited as brown). A final score was then calculated by multiplying the above two scores. If the final score was $\geq 4$, the tumor was considered to have high expression; otherwise, the tumor was considered to have low expression (17).

Reverse-transcriptase PCR (RT-PCR). Cells were washed twice with cold PBS and were collected by centrifugation at $1,000 \times \mathrm{g}$ for $5 \mathrm{~min}$. Total RNA was extracted using TRIzol ${ }^{\circledR}$ reagent (Invitrogen) following the manufacturer's protocol. cDNA was generated with oligo(dT) primers. Primers were designed online using the Primer3 designer program (website, http://frodo.wi.mit.edu/) and were as follows: VMP1 forward, 5'-GAC CAG AGA CGT GTA GCA ATG-3' and reverse, 5'-ACA ATG CTT TGA CGA TGC CAT AA-3'; GAPDH (housekeeping gene) forward, 5'-GGA GAT TGT TGC CAT CAA CG-3' and reverse, 5'-TTG GTG GTG CAG GAT GCA TT-3'. The reaction systems consisted of $0.2 \mu \mathrm{l}$ cDNA, $2.5 \mu \mathrm{l}$ 10X Taq enzyme buffer, $1 \mu 110 \mathrm{~mol} / \mathrm{l} \mathrm{dNTP}$, the forward and reverse primers were $0.2 \mu 1$ and $0.2 \mu 1$ Taq enzymes. Water was then added to $25 \mu \mathrm{l}$. PCR conditions were as follows: denaturation at $95^{\circ} \mathrm{C}$ for $2 \mathrm{~min}$; each cycle consisting of $1 \mathrm{~min}$ at $94^{\circ} \mathrm{C}$, $1 \mathrm{~min}$ at $56^{\circ} \mathrm{C} ; 1 \mathrm{~min}$ and $15 \mathrm{sec}$ at $72^{\circ} \mathrm{C}$; and a final extension of $5 \mathrm{~min}$ at $75^{\circ} \mathrm{C}$. After the reaction, the PCR products were separated on $2 \%$ agarose gel (BioWest) and visualized under UV light.

Xenograftmodel.Mice were treated in accordance with the NIH Guide for the Care and Use of Laboratory Animals (18), and as approved by the Ethics Committee of Ningbo University. Mice were housed in a temperature-controlled room with a proper dark-light cycle, fed a regular diet, and maintained under the care of the Laboratory Animal Unit, Ningbo University, China. 
A
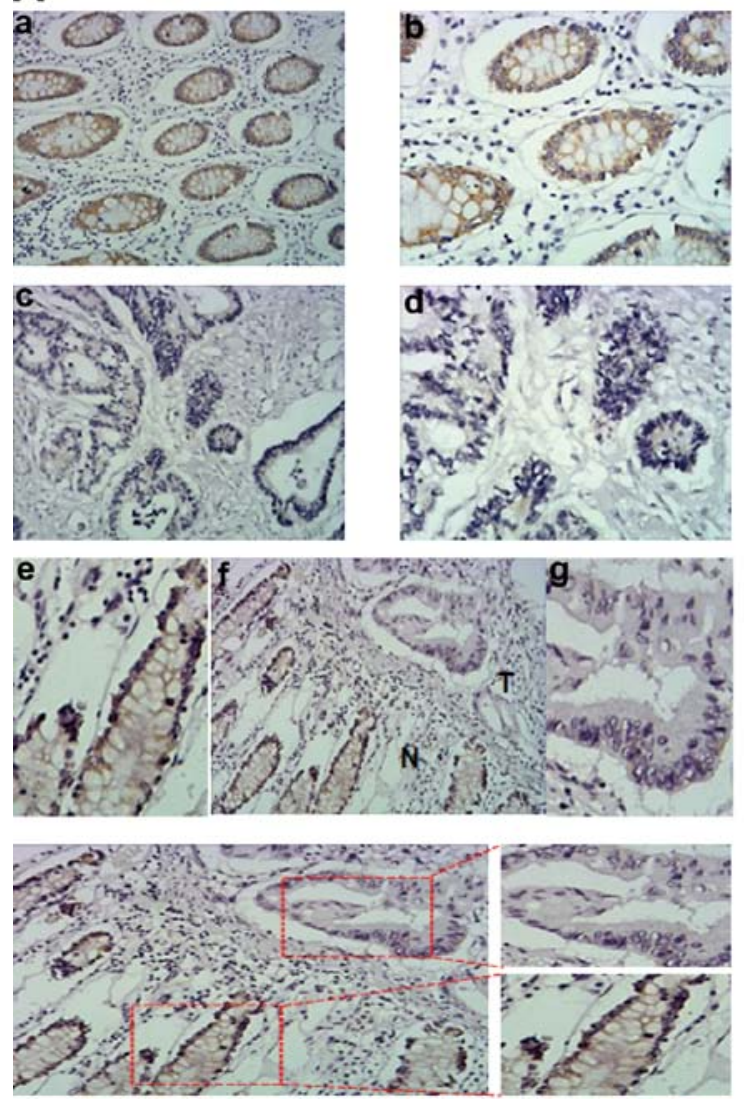

B
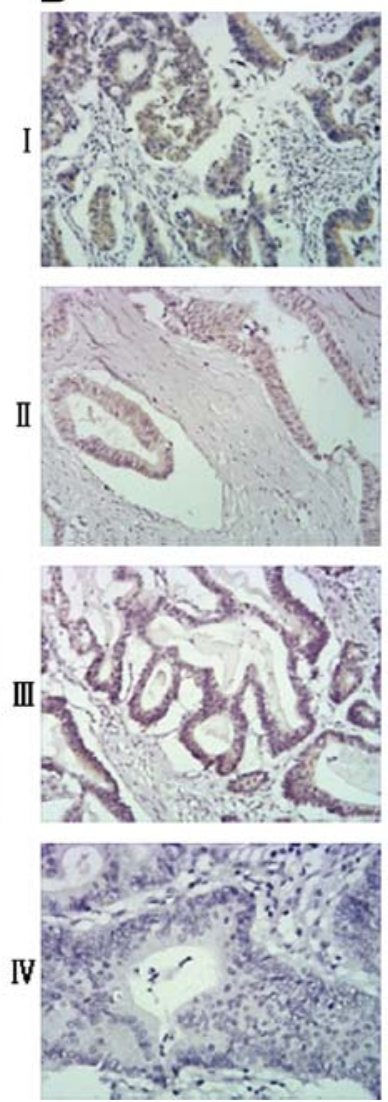
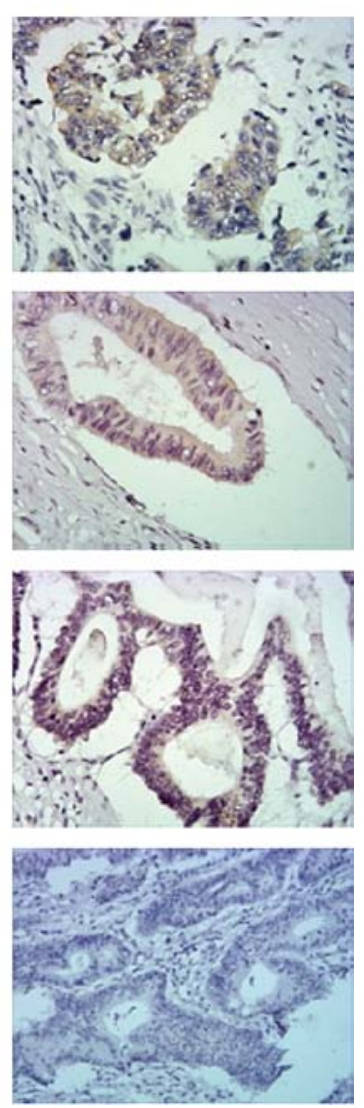

Figure 1. (A) Expression analysis of VMP1 protein by immunohistochemistry. VMP1 expression was mainly localized within the cytoplasm of the cells. VMP1 had strong cytoplasmic staining in the matched adjacent non-cancer tissues (a, x100; xb, 400), weak cytoplasmic or no positive staining in the primary colorectal cancer tissues obtained from the same patients (c, x100; $\mathrm{d}, \mathrm{x} 400)$. VMP1 had increased expression in the adjacent non-cancer tissues compared with that in the colorectal cancer tissues (f, x100; e and $\mathrm{g}, \mathrm{x} 400$ ). (B) The relationship between the expression of VMP1 protein and the stage of the colorectal cancer patients. The results showed that higher staging correlated with lower VMP1 expression.

The mice were acclimated for 1 week before the experiment. $\mathrm{VMP} 1^{\mathrm{KD}}$ and VMP1 $1^{\text {scramble }}$ cells growth in logarithmic phase were trypsinized, and the cell suspension was harvested. After staining with trypan blue, the cells were counted under a hemocytometer to test the viability. Cell concentration was adjusted with culture medium to $1 \times 10^{7}$ cells $/ \mathrm{ml}$. The nude mice were randomly divided into 2 groups. In the VMP1 knockout group, 10 mice were intraperitoneal injected with VMP1 ${ }^{\mathrm{KD}}$ cells, while in the scramble shRNA group, 10 mice were injected with the VMP1 $1^{\text {scramble }}$ cells. Each nude mouse was inoculated with $0.5 \mathrm{ml}$ of the cell suspension. The needle was stopped internally for $5 \mathrm{sec}$, rotated and pulled out to avoid leakage of the cell suspension. The activity, diet and mental state of the nude mice were observed daily. On day 20 of the experiment, all of the mice were sacrificed by cervical vertebra disjointing, the abdominal cavity was opened and the nodules within the abdominal were stripped, counted and confirmed by fluorescence microscopy.

Statistical analysis. All statistical analyses were carried out using the SPSS 13.0 statistical software package. The $\chi^{2}$ test was employed to evaluate differences in expression of VMP1 between the two categories of tissues. The Mann-Whitney $\mathrm{U}$ test was used to analyze the relationship between VMP1 expression and clinicopathological characteristics. Survival curves were plotted by the Kaplan-Meier method and compared by the log-rank test. The significance of various variables for survival was analyzed by the Cox proportional hazards model in the multivariate analysis. $\mathrm{P}<0.05$ in all cases was considered to indicate a statistically significant result.

\section{Results}

Expression of VMP1 in colorectal cancer and analysis of prognosis. Immunohistochemical assay showed that the expression of VMP1 in the same patients was significantly decreased in primary cancer tissues when compared with that in the matched adjacent non-cancerous tissues $\left(\chi^{2}=12.962, \mathrm{P}=0.008\right.$; Fig. 1). Among the matched adjacent non-cancerous tissues, $61.5 \%$ (32 of 52) of the individual tissues had either high or strong VMP1 expression (Fig. 1Aa, b, e and g). However among the colorectal cancer cases only $30.9 \%$ (29 of 94) of the cancer tissues were defined as having high expression of VMP1 (Fig. 1Ac-f). The subcellular location of VMP1 was mainly cytoplasmic.

Correlation between VMP1 protein expression and clinicopathological features. Table I shows the relationship between the expression of VMP1 protein and clinical characteristics. There was no significant correlation between the expression 
Table I. Correlation between the clinicopathological features and the expression of VMP1 protein.

\begin{tabular}{|c|c|c|c|}
\hline \multirow[b]{2}{*}{ Characteristics } & \multicolumn{2}{|c|}{ VMP1 expression } & \multirow[b]{2}{*}{ P-value } \\
\hline & Low & High & \\
\hline \multicolumn{4}{|l|}{ Age (years) } \\
\hline$\leq 60$ & $26(81.3)$ & $6(18.8)$ & \multirow[t]{2}{*}{0.069} \\
\hline$>60$ & $39(62.9)$ & $23(37.1)$ & \\
\hline \multicolumn{4}{|l|}{ Gender } \\
\hline Male & $32(68.1)$ & $15(31.9)$ & \multirow[t]{2}{*}{0.826} \\
\hline Female & $33(70.2)$ & $14(29.8)$ & \\
\hline \multicolumn{4}{|l|}{ Stage } \\
\hline I & $11(57.9)$ & $8(42.1)$ & \multirow[t]{4}{*}{0.008} \\
\hline II & $19(55.9)$ & $15(44.1)$ & \\
\hline III & $30(85.7)$ & $5(14.3)$ & \\
\hline IV & $5(83.3)$ & $1(16.7)$ & \\
\hline \multicolumn{4}{|l|}{ Histological type } \\
\hline $\begin{array}{l}\text { Mucinous } \\
\text { adenocarcinoma }\end{array}$ & $54(72.0)$ & $21(28.0)$ & \multirow[t]{3}{*}{0.266} \\
\hline $\begin{array}{l}\text { Columnar } \\
\text { adenocarcinoma }\end{array}$ & $7(53.8)$ & $6(46.2)$ & \\
\hline Others & $4(66.7)$ & $2(33.3)$ & \\
\hline \multicolumn{4}{|l|}{$\begin{array}{l}\text { Histological } \\
\text { differentiation }\end{array}$} \\
\hline Well & $4(40.0)$ & $6(60.0)$ & \multirow[t]{3}{*}{0.058} \\
\hline Moderate & $46(70.8)$ & $19(29.2)$ & \\
\hline Poor & $15(78.9)$ & $4(21.1)$ & \\
\hline \multicolumn{4}{|c|}{ Tumor diameter (mm) } \\
\hline$\leq 50$ & $46(68.7)$ & $21(31.3)$ & \multirow[t]{2}{*}{0.872} \\
\hline$>50$ & $19(70.4)$ & $8(29.6)$ & \\
\hline \multicolumn{4}{|l|}{ pT classification } \\
\hline $\mathrm{T} 1-\mathrm{T} 2$ & $20(64.5)$ & $11(35.5)$ & \multirow[t]{2}{*}{0.500} \\
\hline T3-T4 & $45(71.4)$ & $18(28.6)$ & \\
\hline \multicolumn{4}{|l|}{ pN classification } \\
\hline No & $30(56.6)$ & $23(43.4)$ & \multirow[t]{3}{*}{0.002} \\
\hline N1 & $25(83.3)$ & $5(16.7)$ & \\
\hline $\mathrm{N} 2$ & $10(90.9)$ & $1(9.1)$ & \\
\hline \multicolumn{4}{|l|}{ pMetastasis } \\
\hline Yes & $5(83.3)$ & $1(16.7)$ & \multirow[t]{2}{*}{0.442} \\
\hline No & $60(68.2)$ & $28(31.8)$ & \\
\hline
\end{tabular}

The data in brackets in Table I are the percentage for each category. VMP1, vacuole membrane protein 1 .

level of VMP1 protein and age, histological classification, histological differentiation, tumor diameter, pT classification or distant metastasis of the colorectal cancer patients. However, the expression of VMP1 was closely associated with stage of the colorectal cancer patients $(\mathrm{P}=0.008)$ and $\mathrm{pN}$ classification $(\mathrm{P}=0.002)$. The expression of VMP1 protein was negatively correlated with staging and $\mathrm{pN}$ classification (Table II). As

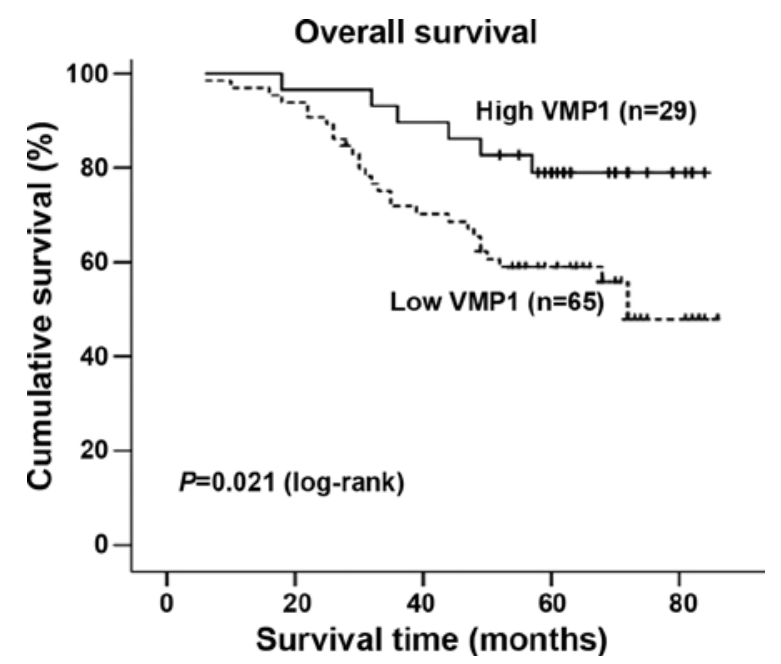

Figure 2. Relationship between the expression of VMP1 protein and patient survival time. Kaplan-Meier curves with univariate analyses (log-rank) for patients with low VMP1-expressing (dotted line) vs. high VMP1-expressing tumors (bold line). The median survival of patients with low VMP1 expression was much shorter (55 months) than those with high VMP1 expression (62 months) ( $\mathrm{P}=0.021$, log-rank).

shown in Fig. 1B, higher staging was correlated with lower VMP1 expression.

Survival analysis. Kaplan-Meier analysis and the log-rank test were used to calculate the effect of classic clinicopathological characteristics (including stage, Tclassification, Nclassification) and VMP1 expression on survival. The expression level of VMP1 protein in the colorectal cancer cases was significantly correlated with the patient survival time $(\mathrm{P}=0.021)$, indicating that lower levels of VMP1 expression were correlated with shorter survival time. The high VMP1 expression group had better survival, whereas the low VMP1 expression group had shorter survival (Fig. 2). The median survival of patients with high VMP1 expression was much longer (62 months) than the median survival of patients with low VMP1 expression (55 months) ( $\mathrm{P}=0.021, \log$-rank).

In addition, $\mathrm{T}$ classification, $\mathrm{N}$ classification and stage were also significantly correlated with survival in the Kaplan-Meier analysis and log-rank test (for $\mathrm{T}$ classification, $\mathrm{P}=0.026$; for $\mathrm{N}$ classification, $\mathrm{P}<0.001$; for stage, $\mathrm{P}<0.001$ ). We did multivariate survival analysis, which included VMP1 expression level, stage, $\mathrm{T}$ classification and $\mathrm{N}$ classification, to determine whether VMP1 expression is an independent prognostic factor of patient outcome. In this analysis, stage was recognized as an independent prognostic factor, but VMP1 expression was not an independent prognostic factor of outcome (Table II).

Expression of VMP1 in different colorectal cell lines. The expression of VMP1 at the protein and mRNA levels in different colorectal cancer cell lines was detected. A negative correlation between VMP1 expression and metastasis was found. Namely, the cell lines expressing VMP1 had a significantly lower ability for metastasis and vice versa. These results suggest that VMP1 may act as a key factor for preventing the progression of colorectal cancer as well as in balancing the level between autophagy and apoptosis (Fig. 3). 
Table II. Univariate and multivariate analyses of different prognostic parameters in patients with colorectal cancer by Cox-regression analysis.

\begin{tabular}{|c|c|c|c|c|c|c|}
\hline & \multicolumn{2}{|c|}{ Univariate analysis } & \multicolumn{4}{|c|}{ Multivariate analysis } \\
\hline & No. of pts. & P-value & Regression coefficient (SE) & P-value & $\mathrm{RR}$ & $95 \% \mathrm{CI}$ \\
\hline Stage & & $<0.001$ & $1.316(0.292)$ & $<0.001$ & 3.729 & $2.103-6.612$ \\
\hline I & 19 & & & & & \\
\hline II & 34 & & & & & \\
\hline III & 35 & & & & & \\
\hline IV & 6 & & & & & \\
\hline pT classification & & 0.026 & $0.502(0.424)$ & 0.236 & 1.653 & $0.720-3.791$ \\
\hline $\mathrm{T} 1-\mathrm{T} 2$ & 31 & & & & & \\
\hline $\mathrm{T} 3-\mathrm{T} 4$ & 63 & & & & & \\
\hline pN metastasis & & $<0.001$ & $0.275(0.443)$ & 0.535 & 1.317 & $0.552-3.140$ \\
\hline No & 53 & & & & & \\
\hline N1 & 30 & & & & & \\
\hline $\mathrm{N} 2$ & 11 & & & & & \\
\hline VMP1 expression & & 0.021 & $-0.496(0.462)$ & 0.283 & 0.609 & $0.246-1.507$ \\
\hline Low & 65 & & & & & \\
\hline High & 29 & & & & & \\
\hline
\end{tabular}

pts., patients; RR, relative risk; CI, confidence interval; VMP1, vacuole membrane protein 1.

A

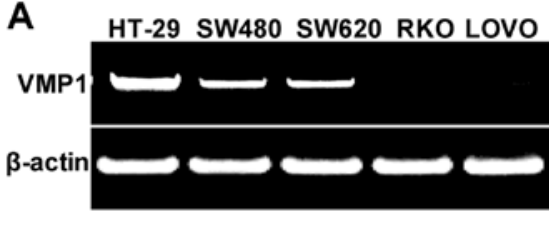

mRNA level of VMP1

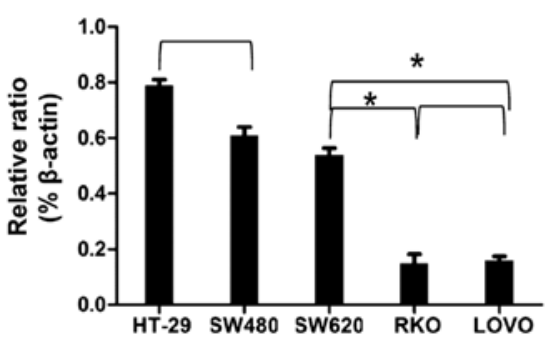

B
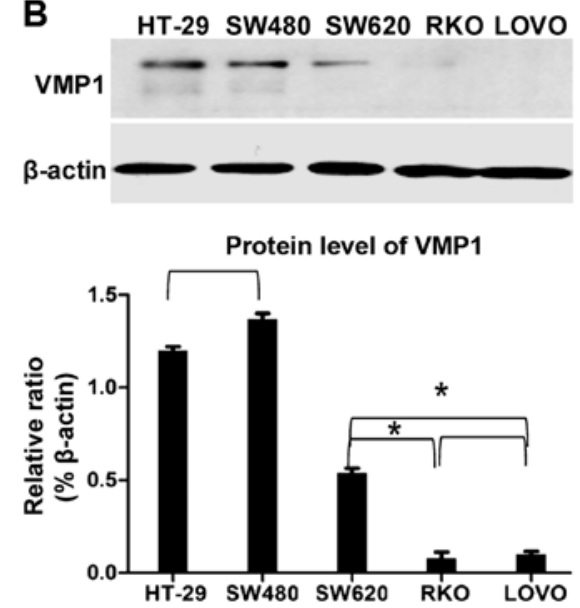

Figure 3. VMP1 protein and mRNA expression levels in different colorectal cancer cell lines. (A) The mRNA expression was determined by RT-PCR analysis and bands were quantified by densitometry, normalized to GAPDH levels. All data are represented as means \pm SD. For each independent experiment, $\mathrm{n}=3$, ${ }^{*} \mathrm{P}<0.05$. (B) Western blotting showed the protein level of VMP1. All values were normalized to that of the control. All data are represented as means \pm SD. For each independent experiment, $\mathrm{n}=3,{ }^{*} \mathrm{P}<0.05$.

Construction and identification of VMP1 stable gene silencing. In order to clarify the role of VMP1 in the progression of colorectal cancer, a shRNA lentiviral vector was constructed to silence the VMP1 gene for heritable changes. We designed 3 pairs of shRNAs. After sequencing was confirmed and amplification in the plasmid, we packaged the 293T cells with liposome and plasmid for a transfection of $48 \mathrm{~h}$. After that, the lentivirus in the culture supernatant was harvested to infect the target cells. Results of fluorescence (Fig. 4A) showed that the cell infection rate reached $\sim 98 \%$. In contrast, the western blotting indicated that shRNA1 was the best sequence for silencing VMP1 at nearly 95\% (Fig. 4B and C). Thus, the colorectal cancer cells were used for subsequent experiments.

Effect of VMP1 gene silencing on cell proliferation and drug sensitivity. First, we evaluated the cell proliferation after VMP1 silencing, which indicated a significantly higher proliferation rate at $48 \mathrm{~h}$ and a balanced level at $72 \mathrm{~h}$ compared with the scrambled shRNA group (Fig. 5A). As shown in Fig. 5C, determination of drug sensitivity indicated an increasing sensitivity 
A
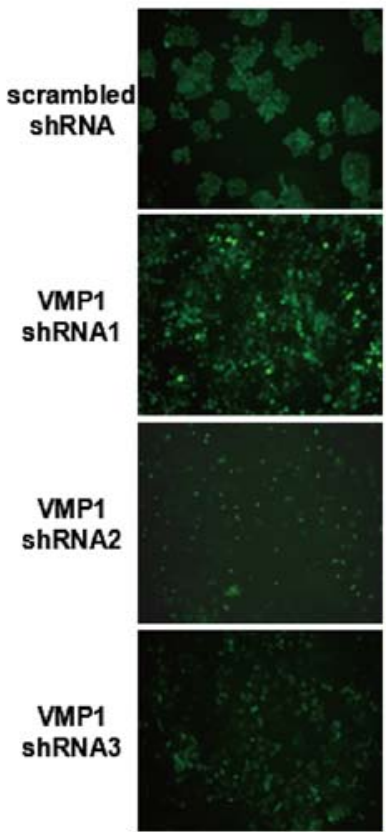

Bright Field
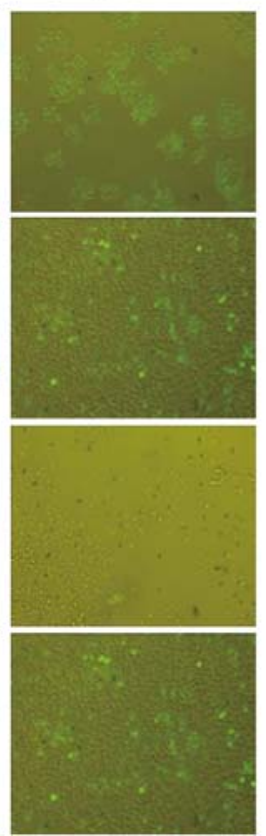

B

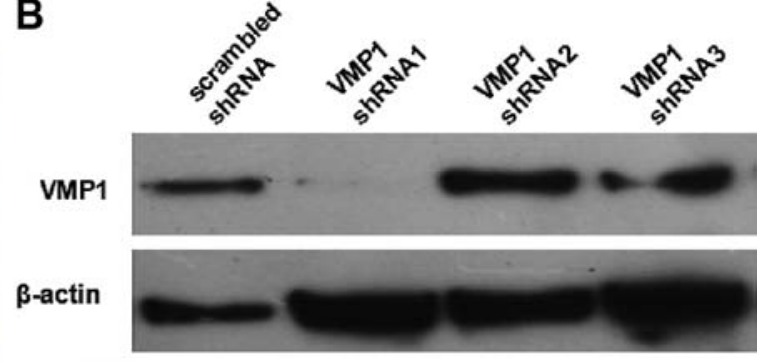

C

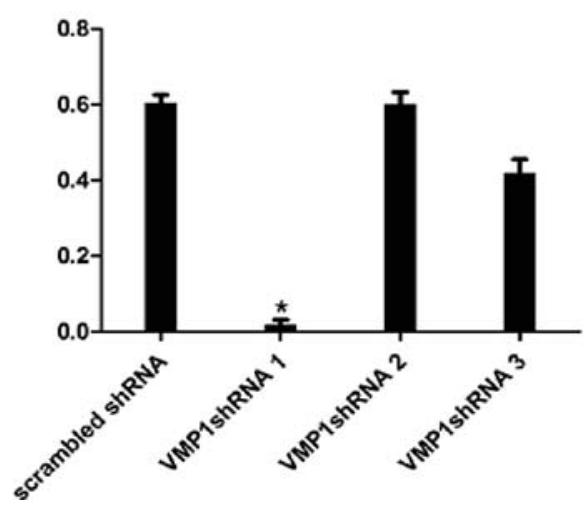

Figure 4. Construction of the VMP1 shRNA lentiviruses and the identification of the gene silencing effect. (A) SW480 cells were infected with the three VMP1 shRNAs and the scramble shRNA virus after $24 \mathrm{~h}$. The left panels show the schematic diagrams under the green fluorescence channel, the right panels show the images under the bright field condition and green fluorescence channel. The fluorescence efficiency of shRNA1 was highest. (B) SW480 VMP1 expression after infection with the viruses was detected by western blotting. (C) Bands were quantified by densitometry and VMP1 shRNA1 gene silencing effect was best; the knockout efficiency was almost 99\% ("P<0.05).

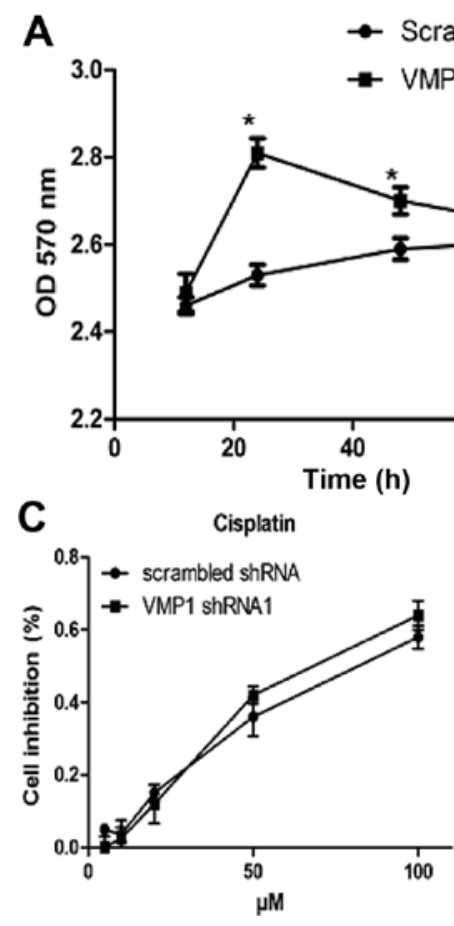

crambled ShRNA

\section{B}

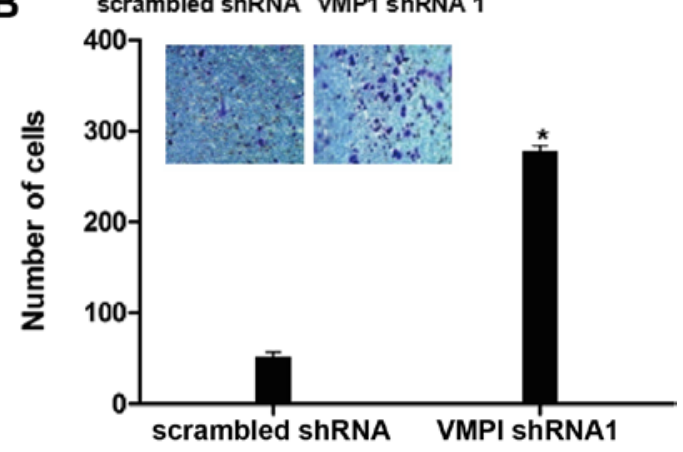

C
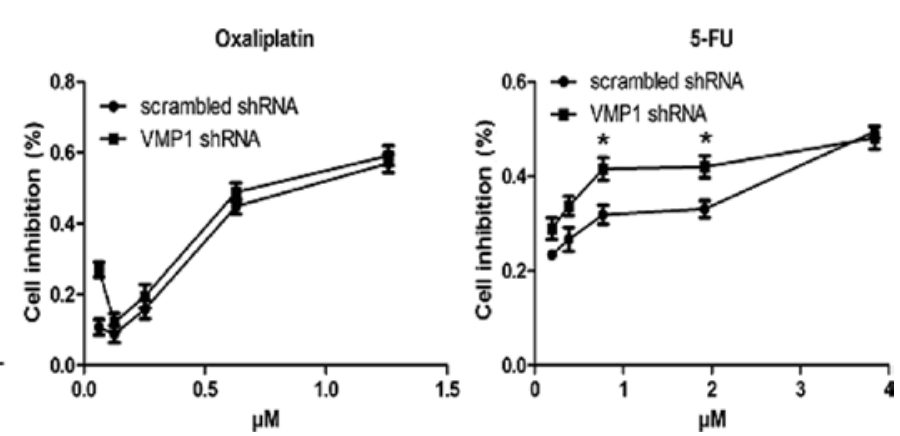

Figure 5. Effects on cell proliferation, invasion ability and drug susceptibility after VMP1 knockout. (A) Cells viability was determined by MTT assay at four different time points, and the cell growth curve was established. Compared with the control group (scrambled shRNA), the proliferation rate of the SW480 VMP1 gene silenced cells (VMP1 shRNA1) was obviously increased $\left({ }^{*} \mathrm{P}<0.05\right)$. (B) The results of the invasion assay. Compared with the control group, the invasive ability was enhanced significantly after VMP1 knockout. Upper left panels show the schematic diagram after the crystal violet staining in the Transwell experiment. Histogram shows the number of cells of the two groups, respectively. (C) The sensitivity of SW480 cells to cisplatin, oxaliplatin and 5-FU before and after VMP1 knockout $\left({ }^{*} \mathrm{P}<0.05\right)$.

to 5-FU after VMP1 silencing but an unconspicuous function on platinum, which resulted from the increased sensitivity to apoptosis signaling.
The effect and mechanisms of cell migration after VMP1 gene silencing. We used a Transwell chamber model to study the ability of cell migration. As expected, a significant increase in 

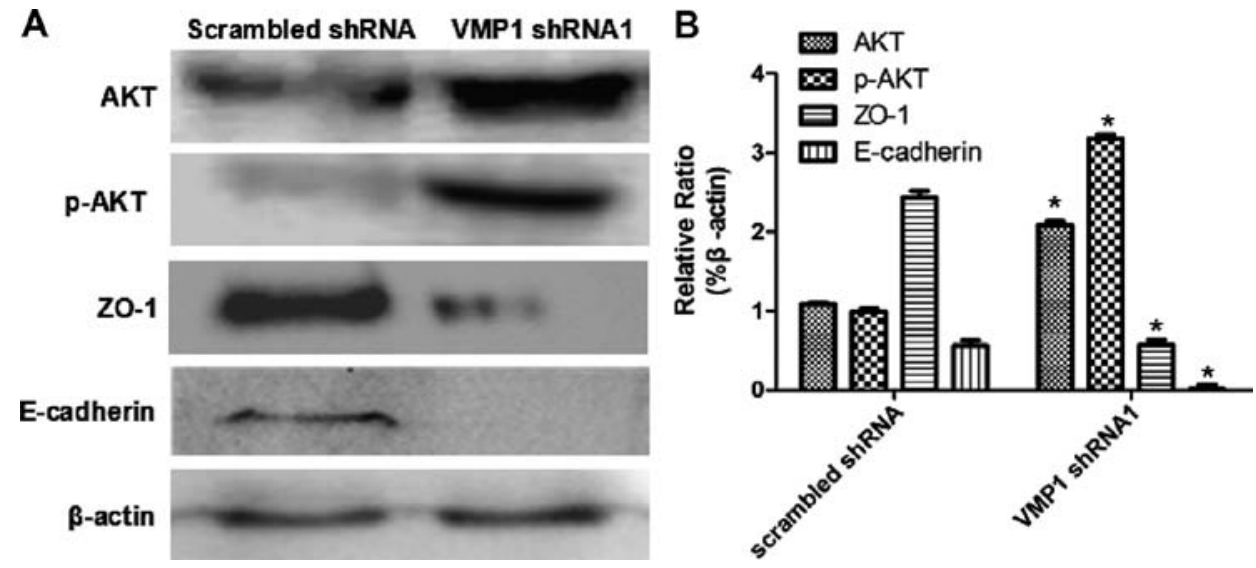

Figure 6. The molecular mechanisms of VMP1 involved in the proliferation and invasion of colon cancer. (A) Protein from the VMP1 shRNA1 and scramble shRNA cells was collected. Akt/p-AKT (involved in cell proliferation) and ZO-1/E-cadherin (related to cell proliferation) protein and $\beta$-actin (used as internal control) were detected by western blotting. (B) Bands were quantified by densitometry. For each independent experiment, $\mathrm{n}=3,{ }^{*} \mathrm{P}<0.05$.
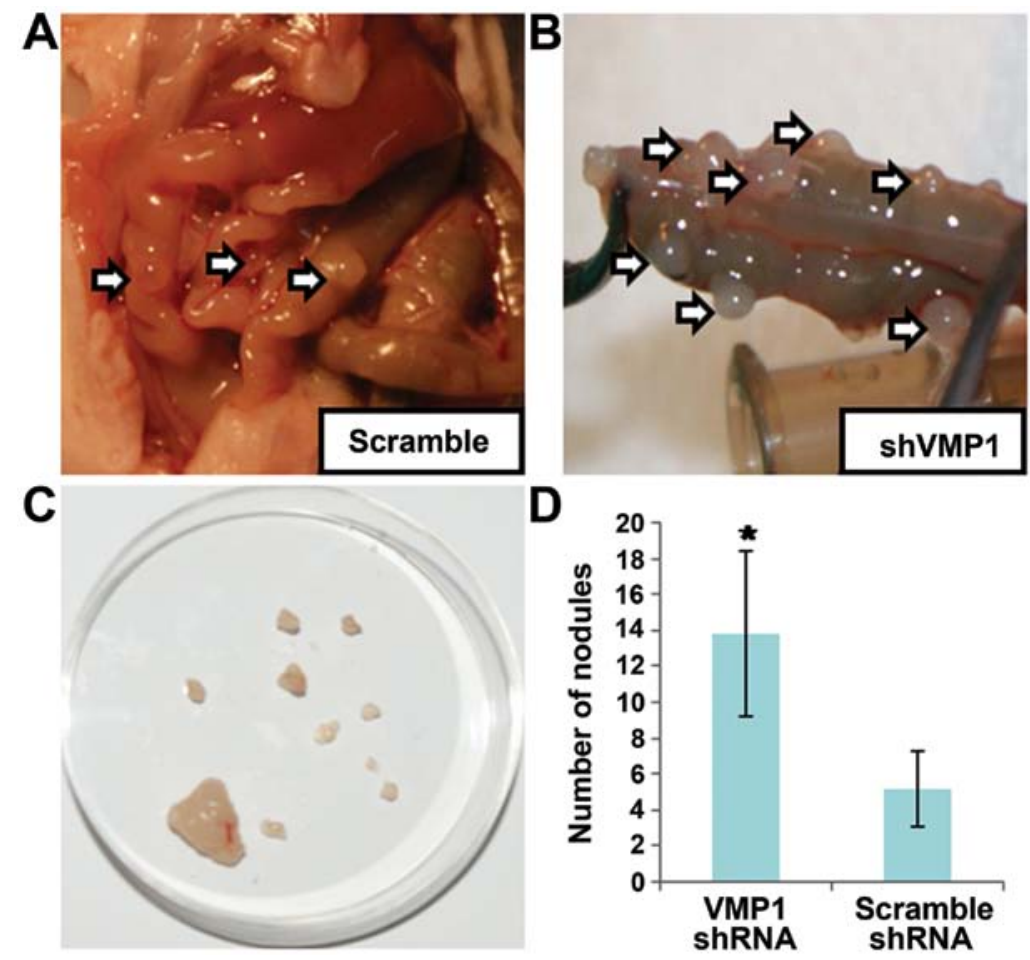

Figure 7. Downregulation of VMP1 promotes tumorigenesis and metastasis of SW480 cells in vivo. (A) In the control group, the number of tumor nodules was less and they were smaller. (B) In the VMP1 gene silenced group, there were many scattered tumor nodules in the greater omentum and mesentery, tumor nodules were pale, hard and not of uniform size. (C) Tumor nodules were stripped and the numbers were counted. The nodular tissues were confirmed by fluorescence microscope with GFP marked green fluorescence. (D) VMP1 shRNA group was 13.9 \pm 4.6 , scrambled shRNA group was 5.2 $\pm 2.1 ;$ the number in the VMP1 shRNA group was significantly more than that in the control group, ${ }^{*} \mathrm{P}<0.05$.

migration was found after VMP1 silencing (Fig. 5B). Western blotting showed an increase in AKT/p-AKT as well as a downregulation of $\mathrm{ZO}-1$, which was related to the activation of the PI3K/AKT signaling pathway and the phosphorylation of ZO-1. In addition, expression of E-cadherin was also downregulated at the same time that acts as a key factor inducing the EMT of tumor cells and promoting migration (Fig. 6).

Biological characteristics of the VMP1 silenced cell lines in vivo. Finally, we compared the tumor formation in a nude mouse model. The results indicated that the tumor formation rate was $100 \%$. There were no significant differences among the two groups of mice at day 7 after inoculation of the normal SW480 cell and the silenced ones. Furthermore, listlessness, poor appetite and slow action were found in the VMP1 shRNA group but no significant changes were noted in the scrambled shRNA group 7 days later. In addition, 20 days after inoculation, separation of nodules surrounded by intestinal tissue was carried out, to evaluate the biological characteristics by counting the nodules with fluorescent signaling. An increasing number of nodules was formed in the VMP1 silenced group compared with the GFP groups (Fig. 7). 


\section{Discussion}

Vacuole membrane protein 1 (VMP1) is associated with cancer patient prognosis which was first observed in studies of pancreatic cancer. VMP1 was found to be overexpressed in pancreatic cancer, and was found to be closely linked with poorly differentiated human pancreatic cancer (13). In addition, zymophagy, a new pathway through VMP1-USP9x-p62 preventing cell death of the pancreas, was reported (12). Furthermore, a study of lung cancer patients found that expression of VMP1 was positively related to poor prognosis (19). However, some studies have shown that VMP1 has various antitumor properties, increasing the degree of malignancy if absent. VMP1 is an important component of cell connections between cells and the formation of tight junctions, and enhances cell junctions and cell adhesion function. In research on breast cancer and renal cell carcinoma, researchers found that VMP1 expression was significantly reduced in cancer metastatic when compared to primary tumors. When VMP1 expression was downregulated, cell adhesion was lost and the invasive capacity of cells increased (20). Another expression study in breast cancer found that patients with VMP1-positive expression had favorable prognosis, and patients with negative expression had poor prognosis (21). Liver cancer studies found similar results. Patients with low VMP1 expression had lower overall survival and disease-free survival than those with high expression of VMP1. Upregulation of VMP1 not only inhibited the proliferation of cancer cells, but also inhibited liver cancer cell metastasis in nude mice (11). The results of the present study and the results in breast and liver cancer are in accordance. Prognosis of patients with VMP1 high expression was significantly prolonged when compared to patients with low expression and the difference was statistically significant. Yet, our results also found that patients with different stages had VMP1 expression level differences. In addition, these results were consistent with previous findings.

VMP1 had a high positive rate in normal tissues and a low positive rate in cancer tissues. In the same patient, VMP1 was positive in the tissue adjacent to the carcinoma tumor tissue, but was not positive in the tumor tissue. Furthermore, a recent study showed that VMP1 was located in the plasma membrane playing a critical role in cell-cell contact (20). It is well known that reduced levels of cell-cell adhesion proteins often correlate with tumor invasion and metastasis (22). Guo et al and other studies have shown that upregulation of VMP1 inhibits metastasis of tumor cells (11). However, to date, no studies have shown the impact of VMP1 in colorectal metastases. Therefore, our results indicated that the development of colorectal cancer may have a negative relationship with the expression of VMP1, which may initiate autophagy and limit the transformation of cancer cells. The results from our study also strengthen the connection between autophagy and cancer metastasis.

In order to further illustrate the concepts mentioned above, an shRNA lentiviral vector was constructed to silence the VMP1 gene to observe heritable changes in cell lines which originally highly express VMP1. After that, the proliferation and migration of shVMP1 cells increased significantly. The benefit of lentiviral vector transfection lies in inducing the cells to gain heritable changes, which benefit subsequent study (23). The traditional method of siRNA interference only functions as a temporary transfection, which affects the proliferation of cells in turn as well as instable function in gene silencing (24). Of course, lentiviral technique also has some defects. It would be time-consuming in the process of puromycin screening in requiring the target cells.

As a tumor-suppressor gene involved in tumor progression, it has been demonstrated that upregulation of VMP1 inhibited the proliferation of liver cancer cells (11). In the present study, after VMP1 knockdown, a maximum proliferation was observed at $24 \mathrm{~h}$ and an ensuing increase which was finally equivalent to the control group. All the mentioned above indicate that VMP1 plays a crucial role in controlling the proliferation of cells. Such a role for VMP1 was further supported by our in vivo study in which we showed that the proliferation of cells was lower than that of the VMP1 gene silenced group.

VMP1 also plays a crucial role in the regulation of cell migration. On the one hand, recent studies indicate that VMP1 is essential for cell-cell contacts and tight junction formation, and it co-localizes with the tight junction protein ZO-1 in spots between neighboring HEK293 cells (20). ZO-1 is important for the clustering of claudins and occludin, resulting in the formation of tight junctions. Downregulation of VMP1 by RNAi results in loss of cell adherence and increased ability for migration. In this process, $\mathrm{ZO}-1$ is closely associated with E-cadherin (25), both of which participate in the course of epithelial-mesenchymal transition (EMT). It was also demonstrated by downregulation of E-cadherin in advanced metastatic colon cancer. In contrast, it was reported that VMP1 may be a new player in the regulation of autophagyspecific phosphatidylinositol 3-kinase complex activation (26). However, the underlying mechanisms involved in the migration of colon cancer remain elusive. To further confirm the subcellular localization of VMP1 and its regulatory mechanism, in our Transwell study, a significant increase in migration was noted in colon cancer cells after VMP1 gene silencing. At the protein levels, a decrease in expression of phosphorylated ZO-1 as well as downregulation of E-cadherin were noted by western blotting, indicating a weak function of ZO-1 after VMP1 gene silencing. As ZO-1 and E-cadherin play important roles in cancer-related cell biological systems (27), the direct interaction of VMP1 with ZO-1 and E-cadherin may provide some evidence on the phenomenon that VMP1 controls colorectal cancer behavior thorough regulation of cell-cell contacts. Moreover, this phenomenon may be associated with activation of the PI3K/Akt signaling pathway, which was embodied by the increasing level of p-Akt/Akt. The PI3K/Akt signaling pathway was confirmed to play a significant role in tumor cell survival, angiogenesis, differentiation, growth and metastasis (28). Similarly, other researches also pointed out the relationship between VMP1 and the PI3K/Akt signaling pathway (29) and the significant role of the PI3K/Akt signaling pathway in colon cancer (30). In the present study, all the findings mentioned above demonstrate the occurrence of EMT and the ensuing cellular invasion may function through the PI3K/Akt signaling pathway.

VMP1 may be a bridge between autophagy and apoptosis. Under conditions of stress, cells may tend to upregulate VMP1 
to promote autophagy. In contrast, when VMP1 is downregulated, the cell may turn to apoptosis. In addition to previous studies in colon cancer (14), in our study, it was demonstrated that selective apoptosis occurred, which was embodied by an increasing sensitivity to 5-FU but an invariable sensitivity to platinum drugs such as cisplatin and oxaliplatin.

Integrated with the above mentioned clinical data, the downregulation of VMP1 caused sensitivity to apoptotic signaling pathways. It will be imperative to discuss the role of VMP1 in in vivo studies, namely to verify the sensitivity to 5-FU of cancer cells with VMP1 gene silencing implanted in animal models. Furthermore, for clinical studies, the effectiveness of 5-FU should be noted in patients with low expression of VMP1, which may be a potential target for individual treatment in colorectal cancer.

In conclusion, the present study firstly indicated that the expression of VMP1 was negatively related to the prognosis of colorectal cancer by analyzing clinical samples and different cell lines. After gene silencing with a lentivirus, a significantly increased ability of invasion was noted and an increased sensitivity to 5-FU was also verified. To some extent, VMP1 could be regarded as a new prognostic biomarker of colorectal cancer and a potential target concerned with migration of cancer cells. In conclusion, VMP1 is not only a bridge in the balance of autophagy and metastasis, but is also a biomarker to predict which patients may benefit from 5-FU in regards to different expression levels of VMP1.

\section{Acknowledgements}

The present study was supported by the Young Medical Talents Training Program of Pudong Health Bureau of Shanghai (no. PWRq2012-31), the Academic Leaders Training Program of Pudong Health Bureau of Shanghai (no. PWRd2012-15), and the Natural Science Foundation of Ningbo (no. 2011A610048).

\section{References}

1. Song J, Guo X, Xie X, Zhao X, Li D, Deng W, Song Y, Shen F, Wu M and Wei L: Autophagy in hypoxia protects cancer cells against apoptosis induced by nutrient deprivation through a Beclin1-dependent way in hepatocellular carcinoma. J Cell Biochem 112: 3406-3420, 2011.

2. Mizushima N: Autophagy: Process and function. Genes Dev 21: 2861-2873, 2007.

3. Debnath J, Baehrecke EH and Kroemer G: Does autophagy contribute to cell death? Autophagy 1: 66-74, 2005.

4. Giatromanolaki A, Koukourakis MI, Harris AL, Polychronidis A, Gatter KC and Sivridis E: Prognostic relevance of light chain 3 (LC3A) autophagy patterns in colorectal adenocarcinomas. J Clin Pathol 63: 867-872, 2010.

5. Koukourakis MI, Giatromanolaki A, Sivridis E, Pitiakoudis M, Gatter KC and Harris AL: Beclin 1 over-and underexpression in colorectal cancer: Distinct patterns relate to prognosis and tumour hypoxia. Br J Cancer 103: 1209-1214, 2010.

6. Miao Y, Zhang Y, Chen Y, Chen L and Wang F: GABARAP is overexpressed in colorectal carcinoma and correlates with shortened patient survival. Hepatogastroenterology 57: 257-261, 2010.

7. Park JM, Huang S, Wu TT, Foster NR and Sinicrope FA: Prognostic impact of Beclin 1, p62/sequestosome 1 and LC3 protein expression in colon carcinomas from patients receiving 5 -fluorouracil as adjuvant chemotherapy. Cancer Biol Ther 14 100-107, 2013.

8. Yang SY and Winslet MC: Dual role of autophagy in colon cancer cell survival. Ann Surg Oncol 18 (Suppl 3): S239, 2011.
9. Kenific CM, Thorburn A and Debnath J: Autophagy and metastasis: Another double-edged sword. Curr Opin Cell Biol 22: 241-245, 2010

10. Dusetti NJ, Jiang Y, Vaccaro MI, Tomasini R, Azizi Samir A, Calvo EL, Ropolo A, Fiedler F, Mallo GV, Dagorn JC, et al: Cloning and expression of the rat vacuole membrane protein 1 (VMP1), a new gene activated in pancreas with acute pancreatitis, which promotes vacuole formation. Biochem Biophys Res Commun 290: 641-649, 2002.

11. Guo L, Yang LY, Fan C, Chen GD and Wu F: Novel roles of Vmp1: Inhibition metastasis and proliferation of hepatocellular carcinoma. Cancer Sci 103: 2110-2119, 2012.

12. Grasso D, Ropolo A, Lo Ré A, Boggio V, Molejón MI, Iovanna JL, Gonzalez CD, Urrutia R and Vaccaro MI: Zymophagy, a novel selective autophagy pathway mediated by VMP1-USP9x-p62, prevents pancreatic cell death. J Biol Chem 286: 8308-8324, 2011.

13. Gilabert M, Vaccaro MI, Fernandez-Zapico ME, Calvo EL, Turrini O, Secq V, Garcia S, Moutardier V, Lomberk G, Dusetti N, et al: Novel role of VMP1 as modifier of the pancreatic tumor cell response to chemotherapeutic drugs. J Cell Physiol 228: 1834-1843, 2013.

14. Qian Q, Zhou H, Chen Y, Shen C, He S, Zhao H, Wang L, Wan D and Gu W: VMP1 related autophagy and apoptosis in colorectal cancer cells: VMP1 regulates cell death. Biochem Biophys Res Commun 443: 1041-1047, 2014

15. Hu H, Krasinskas A and Willis J: Perspectives on current tumornode-metastasis (TNM) staging of cancers of the colon and rectum. Semin Oncol 38: 500-510, 2011

16. Root DE, Hacohen N, Hahn WC, Lander ES and Sabatini DM: Genome-scale loss-of-function screening with a lentiviral RNAi library. Nat Methods 3: 715-719, 2006.

17. Reed J, Ouban A, Schickor FK, Muraca P, Yeatman T and Coppola D: Immunohistochemical staining for c-Kit (CD117) is a rare event in human colorectal carcinoma. Clin Colorectal Cancer 2: 119-122, 2002.

18. No authors listed: NIH requests information on care of laboratory animals. Am J Vet Res 67: 204-205, 2006.

19. Zhou SQ, Zhou JH, Deng T, Zeng F, Wang YK and Liu XG: Expression and prognostic value of vacuole membrane protein 1 in non-small-cell lung cancer. Zhonghua Yi Xue Za Zhi 91: 28282831, 2011 (In Chinese).

20. Sauermann M, Sahin O, Sültmann H, Hahne F, Blaszkiewicz S, Majety M, Zatloukal K, Füzesi L, Poustka A, Wiemann S, et al: Reduced expression of vacuole membrane protein 1 affects the invasion capacity of tumor cells. Oncogene 27: 1320-1326, 2008.

21. Liu F, Niu Y, Liu N, Zhang W, Wang SL, Liu H and Zhang TX: Expression of vacuole membrane protein 1 and its prognostic value in invasive ductal carcinoma of the breast. Zhonghua Bing Li Xue Za Zhi 42: 86-89, 2013 (In Chinese).

22. Okegawa T, Li Y, Pong RC and Hsieh JT: Cell adhesion proteins as tumor suppressors. J Urol 167: 1836-1843, 2002.

23. Lattanzi A, Salvagno C, Maderna C, Benedicenti F, Morena F, Kulik W, Naldini L, Montini E, Martino S and Gritti A: Therapeutic benefit of lentiviral-mediated neonatal intracerebral gene therapy in a mouse model of globoid cell leukodystrophy. Hum Mol Genet 23: 3250-3268, 2014.

24. Subramanya S, Kim SS, Manjunath N and Shankar P: RNA interference-based therapeutics for human immunodeficiency virus HIV-1 treatment: Synthetic siRNA or vector-based shRNA? Expert Opin Biol Ther 10: 201-213, 2010.

25. Ando-Akatsuka Y, Yonemura S, Itoh M, Furuse M and Tsukita S: Differential behavior of E-cadherin and occludin in their colocalization with ZO-1 during the establishment of epithelial cell polarity. J Cell Physiol 179: 115-125, 1999.

26. Molejon MI, Ropolo A and Vaccaro MI: VMP1 is a new player in the regulation of the autophagy-specific phosphatidylinositol 3-kinase complex activation. Autophagy 9: 933-935, 2013.

27. Wodarz A and Näthke I: Cell polarity in development and cancer. Nat Cell Biol 9: 1016-1024, 2007.

28. Acosta KB, Tibolla MM, Tiscornia MM, Lorenzati MA and Zapata PD: Recent patents related to phosphorylation signaling pathway on cancer. Recent Pat DNA Gene Seq 5: 175-184, 2011.

29. Molejon MI, Ropolo A, Re AL, Boggio V and Vaccaro MI: The VMP1-Beclin 1 interaction regulates autophagy induction. Sci Rep 3: 1055, 2013.

30. Huang XF and Chen JZ: Obesity, the PI3K/Akt signal pathway and colon cancer. Obes Rev 10: 610-616, 2009. 